DOI: 10.12731/2227-930X-2018-4-66-73

УДК 007. 51

\title{
АНАЛИЗ СТАТИСТИЧЕСКИХ ДАННЫХ И ПРОГНОЗИРОВАНИЕ КАЧЕСТВА ПОДГОТОВКИ БАКАЛАВРОВ (НА ПРИМЕРЕ НАПРАВЛЕНИЯ ПОДГОТОВКИ 09.03.03, ПРОФИЛЬ ПРИКЛАДНАЯ ИНФОРМАТИКА В ЭКОНОМИКЕ НЧИ КФУ)
}

\section{Еремина И.И.}

В научной работе приводятся доводы аналитического описания оценки качества уровня обученности в подготовке ИТ-специалистов в области народного хозяйства с применением квалиметрических методов для. Вниманию читателей предлагается анализ методов оченки уровня обученности, рассматриваются методы математического моделирования во взаимосвязи с объектами управления образовательным прочессом в вузе. определяется недостаточность разработанных методик оченивания уровня сформированности профессиональных компетенций ИТ-специалистов прикладной информатики в экономике определила актуальность проблемь. Объектом исследования определили теоретико-статистический инструментарий математического моделирования образовательного прочесса, направленного на формирование профессиональных компетенций будущих ИТ-специалистов прикладной информатики в экономике в вузе, при этом предметом исследования явилась спецификация математических моделей формирования профессиональной компетентности выпускников ВУЗа, методика математического моделирования образовательного процесса, алгоритмы, основанные на применении статистических инструментов S-критерий тенденций Дюжонкира, Т-критерий Вилкоксона, Критерий Пирсона $\chi^{2}, \varphi$-критерий (угловое преобразование) Фишера, позволяющие оченить уровень сформированности профессиональных компетенций бу- 
дущих ИТ-специалистов прикладной информатики в экономике и провести временное прогнозирование данных. В иелом представленное для обсуждения исследование имело иель: научно обосновать анализ результатов формирования профессиональной компетентности статистическими методами на примере направления 09.03.03 Прикладная информатика в экономике в Набережночелнинском институте КФУ (НЧИ КФУ).

Ключевые слова: квалиметрия; оченивание; модель оченивания качества уровня обученности; количественные критерии; качество подготовки бакалавров ИТ-направления; трендовый и факторный анализ; прогностическая модель; методы математического моделирования.

\section{STATISTICAL DATA ANALYSIS \\ AND FORECASTING OF QUALITY OF PREPARATION OF BACHELORS (FOR EXAMPLE, AREAS OF TRAINING 09.03.03, PROFILE APPLIED INFORMATICS IN ECONOMICS, NCI KFU)}

\section{Eremina I.I.}

In scientific work arguments of the analytical description of an assessment of quality of level of training in preparation of IT-specialists in the field of national economy with application of qualimetric methods for are given. The readers are offered an analysis of the methods of assessing the level of training, the methods of mathematical modeling in connection with the objects of management of the educational process in the University. the insufficiency of the developed methods of assessing the level of formation of professional competencies of IT-specialists of applied Informatics in the economy has determined the relevance of the problem. The object of the research is the theoretical and statistical tools of mathematical modeling of the educational process aimed at the formation of professional competences of future IT-specialists in applied Informatics in Economics in the University, 
the subject of the study was specification of mathematical models of formation of professional competence of University graduates, methods of mathematical modeling of the educational process, algorithms based on the use of statistical tools S-criterion of the tendencies of Jonker, T-test, Wilcoxon, Pearson's $\chi^{2}, \varphi$-criterion (angular transformation) Fisher, estimating the level offormation of professional competence of future IT-specialists in applied Informatics in Economics and conduct temporary data forecasting. In General, the study presented for discussion was aimed at: scientifically substantiating the analysis of the results of the formation of professional competence by statistical methods on the example of the direction 09.03.03 Applied Informatics in Economics at the Naberezhnye Chelny Institute of KFU (NPI KFU).

Keywords: qualimetry; estimation, model evaluation of the quality of training of students, quantitative criteria; quality of training IT-professionals in Economics, trend and factor analysis; methods of mathematical modeling.

\section{Введение}

Появление потребности в обучении квалифицированных кадров в сфере информационно-коммуникационных технологий (ИТ-специалистов) связано, прежде всего с тем, что информационные ресурсы становятся такими же ценными, как и материальные.

Современная система образования придерживаться двух подходов качеству подготовки выпускников:

1. рассматриваются профессиональные компетентности и технологии их формирования,

2. оценивается уровень обученности рассматриваемых выпускников.

Представленные статистические данные, адаптированные методы математического моделирования, ожидаемые результаты позволяют провести подробный анализ уровеня обученности выпускников экономического отделения, направления «Прикладная информатика в экономике». 
Понятие компетентности и методов ее оценки в настоящее время предложено множество. Однако проблема измерения уровня компетенций (составляющих профессиональную компетентность), формируемых в процессе обучения, до сих пор не имеет общепризнанного решения ни в нашей стране, ни за рубежом. Это связано с тем, что выявляется не однородность субъектов, участвующих в оценке качества образования, что приводит к несогласованности предлагаемых методик и невозможности их системного применения. Следствием этого является отсутствие математических моделей для описания сложного и многогранного понятия «оценки качества», а также методологии измерения уровня качества подготовки студентов в ИТ-сфере. Предлагаемый автором инструментарий позволит оценить качество профессиональной подготовки выпускников вуза и представить ожидаемые результаты исследования этого показателя для поступающих в вузы.

\section{Материалы и методы исследования}

Основной целью исследования является анализ методов оценки качества подготовки ИТ-специалистов в области экономики и рассматриваются методы математического моделирования во взаимосвязи с объектами управления учебно-воспитательным процессом в вузе.

\begin{tabular}{|c|l|}
\hline № & \multicolumn{1}{|c|}{ Задачи исследования } \\
\hline 1. & $\begin{array}{l}\text { обосновать специфику применения квалиметрических методов для } \\
\text { аналического описания оценки качества подготовки ИТ специалистов в } \\
\text { област экономики; }\end{array}$ \\
\hline 2. & $\begin{array}{l}\text { рассмотреть особенности методов математического моделирования, с } \\
\text { целью обнаружения количественных критериев сравнительного анализа } \\
\text { мониторинга учебно-воспитательного процесса в вузе; }\end{array}$ \\
\hline 3. & $\begin{array}{l}\text { предложить количественные критерии описания оценки качества } \\
\text { подготовки, позволяющие получить данные о качестве академической } \\
\text { подготовки ИТ-специалистов в области экономики в учебном заведении; }\end{array}$ \\
\hline
\end{tabular}

Подготовка дипломированных бакалавров по основной профессиональной образовательной программе (ОПОП) по направлению 09.03.03 «Прикладная информатика» ведется в ФГАОУ ВО НЧИ КФУ с 2011 года. 
Для проведения нашего исследования использовались следующие математические методы статистической обработки результатов: общестатистические методы, временное прогнозирование, непараметрические статистические методы (Критерий Пирсона $\chi^{2}$, $\lambda$-критерий Колмогорова-Смирнова, $\varphi$-критерий (угловое преобразование) Фишера, коэффициент ранговой корреляции Спирмена rs), параметрические статистические методы (t-критерий Стьюдента), регрессионно-корреляционный анализ.

\section{Результаты исследования}

Обозначим количественные критерии описания оценки качества подготовки ИТ-специалистов в области экономики, позволяющие получить данные о качестве академической подготовки в учебном заведении:

\begin{tabular}{|l|}
$\mathrm{P}_{\mathrm{ij}}$ - показатель обученности/оценка; \\
\hline $\mathrm{V}_{\mathrm{ij}}$ - показатель уровня академической подготовки по i-му блоку; \\
\hline $\mathrm{S}_{\mathrm{ij}}$ - итоговый показатель. \\
\hline
\end{tabular}

Информационная база исследования содержит данные за четыре года (сентябрь 2014 по февраль 2018). В исследовании участвуют студенты со второго по четвертый курс.

\section{Обсуждение}

Анализируя информационную базу по уровню обученности была построена трендово-факторная прогностическая модель описания оценки качества подготовки ИТ-специалистов в области экономики.

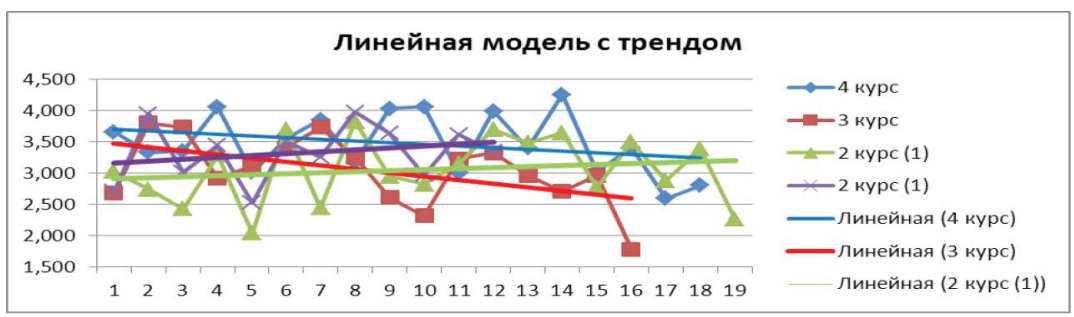

Рис. 1. Линейная модель всех курсов с трендом 

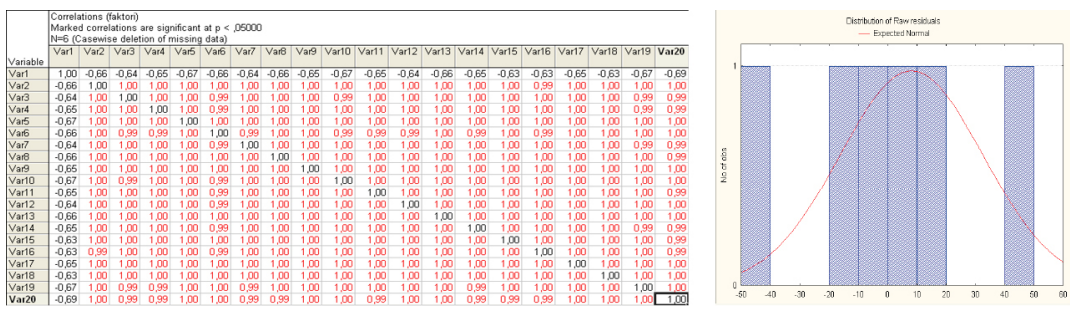

Рис. 2. Множественная регрессия

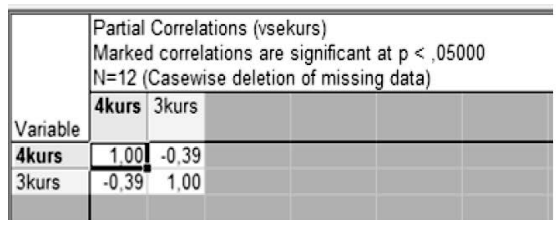

Рис. 3. Парная корреляция 4 и 3 курсов

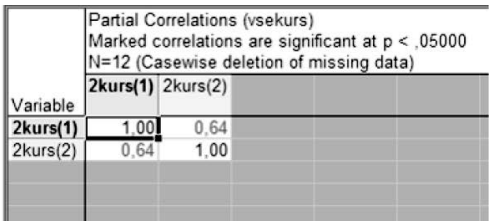

Рис. 4. Парная корреляция 2(1)

и 2(2) курсов

Рисунок 4 показывает связь переменных 2 курс (1) и 2 курс (2), что говорит о необходимости дальнейшего анализа этих переменных.

Построена математическая модель для всех четырех курсов.

Для примера предоставим математическую модель для второго курса:

$\left\{\begin{array}{l}P_{11} V_{1}+P_{12} V_{2}+P_{13} V_{3}+P_{14} V_{4}+P_{15} V_{5}+P_{16} V_{6}+P_{17} 7_{1}+P_{18} V_{8}+\ldots P_{1 n} V_{m} \ldots+P_{125} V_{25}=S_{11} \\ \cdots \ldots . \\ P_{121} V_{1}+P_{122} V_{2}+P_{123} V_{3}+P_{124} V_{4}+P_{125} V_{5}+P_{126} V_{6}+P_{127} 7_{1}+P_{128} V_{8}+\ldots P_{12 n} V_{m} \ldots+P_{1225} V_{25}=S_{121}\end{array}\right\}$

Формула 1. Математическая модель для второго курса

\section{Заключение (выводы)}

Предварительное исследование показало, что наибольшее влияние на повышение уровня обученности ИТ-специалистов оказали такие факторы как материально-техническое обеспечение (использование в процессе обучения вычислительной техники, технические характеристики вычислительной техники, внедрение в учебный процесс интерактивного оборудования и др.), применение в учебном процессе элементов электронного обучения, уровень квалификационного профессионализма профессорско-преподавательского состава. 


\section{Список литературы}

1. Lysanov D.M., Karamyshev A.N., Eremina I.I. Comparative evaluation of quality characteristics of process equipment // Astra Salvensis. 2017. T. 2017. C. 217-224.

2. Дубцова М.М. О реализации квалиметрического подхода в решении проблемы оценивания учебных достижений студентов вуза // Перспективы науки. 2011. №5(20). С. 9-11.

3. Еремина И.И. Квалиметрический подход оценивания качества академической подготовки ИТ-профессионала в вузе // Научная дискуссия: вопросы педагогики и психологии: сборник статей по материалам XXV международной заочной научно-практической конференции. М., Изд. «Международный центр науки и образования», 2014. №4(25). 228 с. С. 137-139.

4. Еремина И.И. Решения класса ERP как средство реализации практикоориентированной междисциплинарной подготовки инженеров, экономистов и менеджеров // European Social Science Journal. 2017. № 11. С. 193-203.

5. Еремина И.И., Макусева Т.Г. Моделирование результатов формирования профессиональной компетенций с использованием среды «STATISTICA 6.0» // Бюллетень науки и практики. 2018. Т. 4. № 11. C. 485-493.

\section{References}

1. Lysanov D.M., Karamyshev A.N., Eremina I.I. Astra Salvensis. 2017. V. 2017, pp. 217-224.

2. Dubtsova M.M. Perspektivy nauki. 2011. №5(20), pp. 9-11.

3. Eremina I.I. Nauchnaya diskussiya: voprosy pedagogiki i psikhologii: sbornik statey po materialam XXV mezhdunarodnoy zaochnoy nauchno-prakticheskoy konferentsii [Scientific discussion: questions of pedagogy and psychology: a collection of articles based on the materials of the XXV international correspondence scientific-practical conference]. M., Izd. «Mezhdunarodnyy tsentr nauki i obrazovaniya», 2014. №4(25), pp. 137-139.

4. Eremina I.I. European Social Science Journal. 2017. № 11, pp. 193-203. 
5. Eremina I.I., Makuseva T.G. Byulleten' nauki i praktiki. 2018. V. 4. № 11, pp. 485-493.

\section{ДАННЫЕ ОБ АВТОРАХ}

Еремина Ирина Ильинична, доцент кафедры Бизнес-информатики и математических методов в экономике кандидат педагогических наук, доцент

Набережночелнинский институт (филиал) ФГАОУ ВО «Казанский федеральный университет»

проспект Мира 68/19 (1/18), г. Набережные Челны, Республика Татарстан, 423812, Российская Федерация ereminaii@yandex.ru

\section{DATA ABOUT THE AUTHORS}

Eremina Irina Il'inichna, Associate Professor of the Department of Business Informatics and mathematical methods in Economics, Candidate of Pedagogical Sciences, Associate Professor Naberezhnye Chelny Institute (branch) of Kazan Federal University” 68/19 (1/18), Mira str., Naberezhnye Chelny, Republic of Tatarstan, 423812, Russian Federation ereminaii@yandex.ru ORCID: 0000-0003-2333-3935 ResearcherID: M-2946-2015 Scopus Author ID: 56498936700 